\title{
Collective Behavior in Wireless Sensor Networks
}

\section{Ryszard Klempous}

\section{Wroclaw University of Technology}

27 Wybrzeze Wyspianskiego street, 50 - 370 Wroclaw, Poland

e-mail: ryszard.klempous@pwr.wroc.pl

\begin{abstract}
The system of homogeneous nodes, cooperating with each other, pursuing strictly defined goals for the exchange of information... this is a Wireless Sensor Network (WSN). WSN's are network radio equipment (transmitters, sensors and microcontrollers), that are geographically distributed in a defined area. These autonomous nodes communicate with each other and jointly submit their data through the network to the Base Station (BS). The basic objective of such activity is to monitor environmental conditions, such as: light, pressure, temperature, motion and/or chemical pollution. This paper describes a relational shape adaptive collective behavior. On the basis of formal set theory, as shown from the perspective of global managed adaptive WSN actions, and how local activity adaptation is guaranteed.
\end{abstract}

Keywords: adaptive systems; collective behavior; Wireless Sensors Network; WSN

\section{Introduction}

Wireless Sensors Networks (WSN's) are important for the following reasons:

- Limited range radio communications nodes naturally breakdown to local and global. This is all that is heard in the environment is for the local node, the rest of the network from the perspective of node WSN is global

- Radio frequency communications nodes, in the common band, provide an effective exchange of information between neighbors. A node, while monitoring communication in the environment continues to monitor the needs and situations of neighbors and can interact with them in the attainment of common objectives.

- Radio communications in a limited range, stimulates group activities. A node, as an entity, performs simple activities and I limited to neighbor 
relationships (and therefore local) jobs. In order to achieve the objective, joint actions for the entire network (global) cooperation is essential in many nodes. Without interaction and group activities the WSN is not justified.

Normally, distributed WSN's integrate and manage a wide range of systems that use low power microcontrollers (or smart sensors), and in addition, are equipped with sensors, radio transmitters and power sources $[6,12]$. The sensors perform regular measurements that monitor, for example, vibration, temperature, humidity and light. Then, the results of the measurements are forwarded to the base station (BS), which is connected to the computer network in which aggregation/synthesis are performed and the final analysis of the data. In isolation, the individual nodes are limited, but become powerful, when numbers integrate into a larger network and collaboration environment. The WSN's are a usual asset of two subsystems; the collection of data and the network broadcast information system [12]. Also, in this case, we will use a layered model network as applied to the ZigBee protocol (IEEE 802.15.4).

\subsection{Network Layer Communication and Routing in WSN}

Usually in hierarchical design communication in a WSN network, a layer is located between the application layer (APS) and MAC $[9,20]$. It provides the MAC layer data services and traffic management services. The transmission of data, in order to determine flow of packets in a network, uses the higher layer mechanisms. It consists of packet header information, including the address, and if this requires the implementation, as well as, authentication and confidentiality codes. The traffic management provides several control functions; it may start setting up new network routing, set up a new device, determine the level of performance/failure of adjacent nodes, and thus decide whether and which of the nodes are adjacent to the shortest path (routing), change diagrams (e.g., unicast to broadcast) and monitor the WSN nodes to determine their usefulness in creating the communication network structure. The higher Layer (application) is helpful when configuring the network layer.

\subsection{A Self Organization and WSN Protocols}

The WSN can often be used in difficult conditions, which results in a high rate of electrical energy consumption within the nodes. In order to ensure, that the WSN will be still effective, certain mechanisms that compensate for energy degradation should be an integral part of design of the WSN [16].

In order to achieve this, the core of the WSN must have very robust routing protocol algorithms that are needed to ensure that the network will be able to refocus itself to make ad hoc or self repairs. When is such a property desirable? Most often this will be in the event of changes in the environment, which will 
reduce performance of the communication networks [15]. Hence an important component design of WSN's is the choice of network protocol, which is matched to the tasks for which the network is intended. There is no single or universal solution. There are different size memory resources for nodes for communication node strategies and that is their complexity. As an example here, we can give ZigBee [20], Wi-Fi, Bluetooth, enOcean, Z-wave and Insteon [25].

\subsection{Capabilities and WSN Limitations}

In order to determine a paths packet flow, most routing protocols in a WSN terminate at the network layer, using exploring/discovering attempts. On the basis those control packets, routing tables are dynamically refreshed. If a known node is not responding, the item is removed from the routing table and node propagates the modified information, in order to reflect observed changes in the network configuration [22].

An intelligent collaboration environment results from the use in each of the component WSN chipset drivers. This approach is crucial for the use of sensors that incorporate microprocessors, after their integration, with a certain form of intelligence [1] or the microprocessor signal alignment systems, which enhance the capabilities. Integration of devices with microcontrollers have brought significant benefits to the technology of sensors, so that now they match signal interfaces, hardware, multicast routes and the opportunities for cooperative networks are increasingly integrated within the sensor. Adding to the sensor microcontroller, in addition, makes it possible to use advanced algorithms, digital signal processing (DSP), leading to faster and more reliable data acquisition systems.

WSN networks cannot guarantee a direct connectivity communication type LineOf-Sight (LOS), we need to try and eliminate any impact of terrain or weather effects on the RF transmission. Oxygen and water are two of the main atmospheric factors filtering in the frequency range $2 \mathrm{GHz}$ to $40 \mathrm{GHz}$. It should therefore be expected to account for these factors on the communication capabilities and routing paths to the base station in the WSN. Signal loss in the atmosphere, and multi-displacement terrestrial communications also takes place, so to find the appropriate routing paths in the network topology is an important factor. Transmission between two nodes may not be maximal due to interference in the band RF and as practice shows, it is not a very important factor. The interference in the band RF very often eliminates a number of nodes shared in communication. For example, Bluetooth communication operates in the $2.4 \mathrm{GHz}$ band. So, if a computer that communicates with peripheral devices wirelessly (e.g. keyboard, mouse, printer, etc.) using this Bluetooth standard and it operates in the same environment as the WSN, the risk of interference is very real. Other sources of interference are harmonics generated by monitors, fluorescent lights or electric motors [20]. 
Density of sensors in the network must be designed with the optimal number of nodes, with the flexibility of accuracy spatial distribution of collected data, as well as to ensure flexible routing decision-making at the time, such as rebuilding route tracks. Comparison to the neighbor devices interval Signal to Noise Ratio (SNR) to make it easier to select your device with higher SNR and therefore find best (cooperating) nodes in the vicinity is the foundation for efficient routing. If the density increases, the sensor spatial distribution increases the level of flexibility of solutions, and to find the best path routing is more likely. In addition, additional nodes in the network reduce network congestion, which ensures the additional benefit of high Quality of Service (QoS).

Usually, sensors need to work in an environment where energy is retrieved from a battery, which is an important constraint on the design and implementation WSN. Because of the spatial distribution and the limited resources available, the activity sensor is reset to the local activities and mutual communication between neighbors. The network communications (local) coordination of activities for the WSN implementation tasks, which one node cannot achieve, is one of the key actions in the network.

Information that is collected by the sensors in a specific area must be forwarded to the BS, however, because of the reduction in power and limited range radio transmission at each node, communication is carried out with the multi-hop. As a result, information may be transmitted by a large area, which significantly exceeds radio coverage one single node. Indiscriminate broadcasting of information does not guarantee success in a WSN, since it takes up bandwidth. Reducing the transmission bandwidth channel, thereby increasing the usable collisions probability in a communications channel, ensures an increase in energy consumption. Individual nodes have limited resources and the flow of information which is required for nodes to the BS must be implemented in a manner that minimizes energy consumption.

\section{Multi-Hop Routing}

There are a considerable number of investigations for routing WSN algorithms, which allow you to transfer information between nodes and a BS [2, 3, 19]. The flat algorithm (e.g. flooding) ensures that information ends at the BS, unless there is no node that can communicate with it directly. Such protocols use broadcast to most techniques and practically entail flooding the WSN data packets. This results in collisions in the communication channel and repeated retransmissions. Approach flooding is not only fully ineffective, but also deteriorates usable bandwidth and restricts communication speed, resulting in poor quality communications, leading in extreme cases, to a cut off in areas of the WSN. These disadvantages are minimized in the GLIDER protocols [7], where communication 
is limited only to broadcast neighborhood nodes. Routing Protocols, such as data Centric Routing for Sensor Protocol for information via Negotiation (SPIN) in implementing communication is geared to minimize the duration of transmissions, the number of additional queries and response times to events recorded by the sensor is at the end of other information requirements.

Rumor routing protocol $[2,23]$ attempts to take advantage of software agents and directed communication mechanisms. In this case, positive results are achieved on a relatively small number of scenarios, in situations where the cost/energy resources are small compared to the costs of communications. Hierarchical protocols, such as LEACH, PEGASIS or TEEN [17, 20, 24, 27] use a directed communication implemented mechanism by creating a hierarchical topology with clusters or divisions by zone. In this paper we to go further, using a routing protocol based on a tree structure so that the chains use a novel energy efficient tracking algorithm called Predict-and-Mesh (PaM) [28, 29, 30], which is suitable for energy sensitive distributed wireless tracking systems. Making use of the PaM algorithm, it is possible to adaptively adjust the sensing frequency for pervasively monitoring various kinds of targets with random movement patterns.

All the above mentioned protocols make use of a single multi-hop communication path, between a node and a BS. The resulting communication path is used as long as it is energy efficient justified $[8,9,10,13]$. If the level of a node, which is one of the tracks, drops below a certain minimum level, path determination algorithms are activated. The newly created track is in operation after the energy resource exhaustion of its elements. This is repeated as long as the algorithm is not able to set a new path. Then we recognize that the WSN ceases to operate.

\subsection{Routing Systems with Retransmission}

Construction track routing protocols in multi-hop neighborhood nodes requires cooperation in order to establish communication routes. Regardless of whether one or multiple paths are designated, routing protocols attempt to construct them so that they lead to the BS. The operation of a single node when routing, although carried out locally in the neighborhood, needs to be underpinned by an objective, that is, to provide global data packet to the base station (BS). Additional criteria, such as cost minimization of energy consumed, to minimize the number of retransmissions, minimize delays, expressed as functions, to help you to choose the appropriate data transfer direction collected in the WSN. For a spatial communication approach, it is slightly different than those described above. Instead of making use of the features, we use relationships. There is no need to (although it is possible for them to do so) make accurate decisions, which node is to be the next in the path to the BS. Instead, what we need is only to create a communication pathway to meet a specific relationship between the successive nodes forming it. Such an approach requires that already in the path are required data packets, nodes cooperate different locally in the neighborhood, based on 
local/current criteria and making decisions locally. However, all of these local measures must be correlated in such a way that the resulting spatial communication meet the criteria set out on a global scale. Developing cooperation within network nodes uses topological properties, but it is not centered on a specific direction - nodes simply cooperate with each other in the vicinity $[10,11]$. However, the entire WSN focuses on the collection of information from the sensors and sends it to BS. Thus, the nodes, which operate in the vicinity, must ensure that the deposit local actions will establish a path through the nodes in the BS.

\section{A Description of the Actions Using Relations}

The proposed method for Multi-hop routing uses three, two-fold, relations defined in activities set (Act) that describe communication activity in the WSN. These three relations are: subordination $(\pi)$, tolerance $(\vartheta)$ and collision $(\chi)$, proposed by Jaron [8]. They are particularly useful in modeling and visualization quality interactions, especially within the WSN [5, 17, 18]. In order to present our solutions, we will utilize the following relationships:

$$
\pi:=\{<x, y>; x, y \in A c t \mid x \pi y\}
$$

An expression $x \boldsymbol{\pi} y$ - defines operation $x$, which is subordinated to $y$, otherwise saying the $y$ dominates the $x$,

$$
\vartheta:=\{<x, y\rangle ; x, y \in A c t \mid x \vartheta y\}
$$

The $\vartheta$ means that the $\mathrm{x}$ and $\mathrm{y}$ are very sensitive to each other,

$$
\chi:=\{<x, y>; x, y \in A c t \mid x \chi y\}
$$

It should be noted that each two-fold relation $R$ defined on a set $A$, can be represented as a set of ordered pairs $\langle\mathrm{x}, \mathrm{y}\rangle$. In set theory, basic properties of our three relationships mentioned above we define [10] as follows:

$$
\begin{aligned}
& \pi \cup \vartheta \cup \chi \subset A c t \times A c t \neq \varnothing, \\
& \imath \cup(\pi \cdot \pi) \subset \pi
\end{aligned}
$$

Where $\imath \subset A c t \times A c t$ is identity on the Action set. Next

$$
\pi \cup \vartheta^{-1} \cup(\vartheta \cdot \pi) \subset \vartheta
$$

Where $\vartheta^{-1}$ means an inverse relationship to $\vartheta$. 
Thus,

$$
\vartheta^{-1}:=\{<x, y>\in X \times Y \mid y \vartheta x\}
$$

For the relation of collision taking place:

$\chi^{-1} \cup(\pi \cdot \chi) \subset \chi \subset \vartheta$

Where $\vartheta$ ' is the relationship $\vartheta$, which is expressed as:

$$
\vartheta^{\prime}:=\{\langle x, y\rangle \in X \times Y|<x, y\rangle \notin \vartheta\}
$$

Axiom (3.8) in relation to collision was separable in relation to tolerance, symmetrical and that the actions were subordinated in collision with all the activities, which is dominant in the collision. In $[14,15,18]$ shown as using these two-fold relations for model spatial communication. The subordination relation can be applied for multi-hop routing path determination; the tolerance allows multiple paths to exist simultaneously while the relation of collision represents some form of conflicts and restrictions in communications space. The relation of subordination allows you to specify the routing path retransmission, while the tolerance permits the existence of many such paths, and relationship conflict creates a limitation required communication space.

\section{Chaining in WSN}

Using the appropriate relationship for chains is, in most cases, describing synergisms between adjacent nodes and adjusting them to the general conditions prevailing in the area. Let's look at the relationship of subordination $(\pi)$. Among all three relationships, only that the only is transitive, this allows us to track modeling retransmission. Relation $(\pi)$ is a transitive and reflexive one and is therefore preorder in the set of Actions.

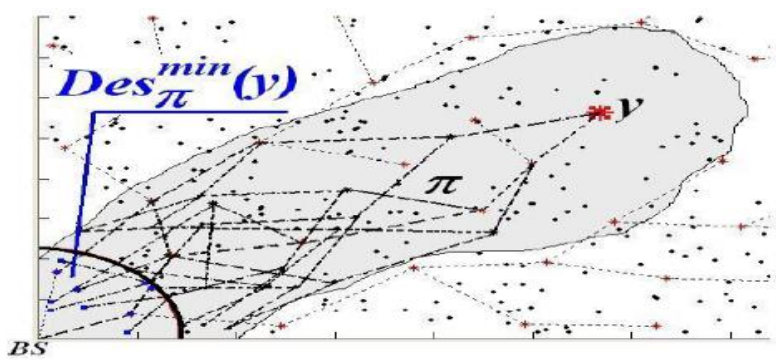

Figure 1

Creating $\operatorname{Des}_{\pi}(y)$ in the WSN [17] 
Further studies, however, require more powerful order in the Actions set. Entering a partial order, which is a particular instance of preorder, does not seem to be difficult. In real-time applications, nodes are distributed more or less randomly in the area. In the case where the two nodes of the network are located very close to each other, one of them is mute remaining in the stand by. This creates a "single" node communication with much greater energy resources. Such a combination of two components can be expressed by applying the anti-symmetric condition on the relationship of subordination. So we have in the set of activities Action defined partial order (weak). Let us go a step further, set Action is partially ordered (weak), but strong and weak relations are closely related in the sense that it's easy to convert one type of relationship to another one. So let's do this, the relationship of subordination is asymmetrical in nature, which is related to its axiomatic antireflexivity. So let's assume that our relationship of subordination will be additionally anti-reflexive. Is this assumption is consistent with the status of the actual WSN network? This seems to be the case. The situation, when a node transmits a packet to itself seems not to be in the category of reasonable behavior. Therefore, eliminating such situations is most appropriate. Reached in this way the relationship of anti-reflexivity is strong partial order in the Actions set. Further, a detailed description of the chain of relationships can be found in [18] and as the final result

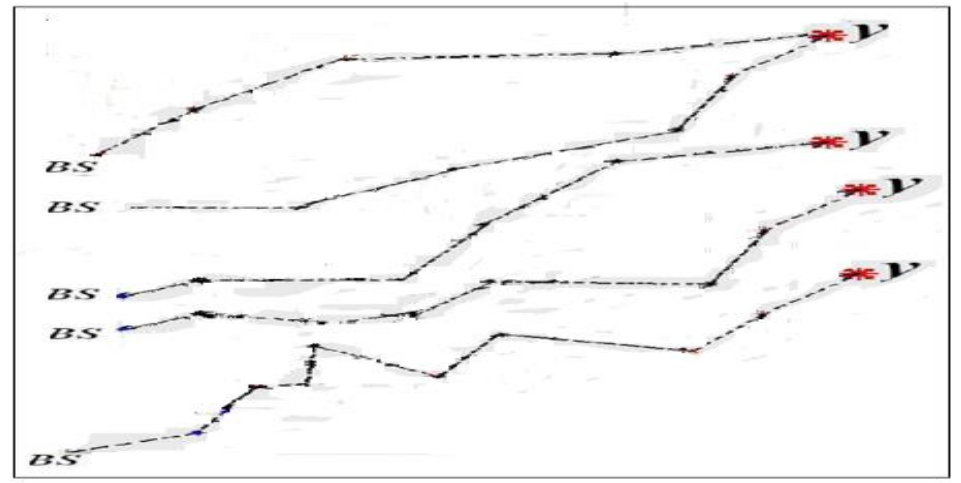

Figure 2

Examples of chains of the $C h n_{\pi}(y)$ set

One obtains four partially ordered sets:

$$
\begin{aligned}
& \left.\operatorname{Des}_{\pi}^{\min }(\mathrm{y})=\left\{\mathrm{x} \in \operatorname{Des}_{\pi}(\mathrm{y}) \mid B S \pi x\right)\right\} \\
& \operatorname{Des}_{\pi}^{\max }(\mathrm{y})=\left\{\mathrm{x} \in \operatorname{Des}_{\pi}(\mathrm{y}) \mid x \pi B S\right\} \\
& \operatorname{Des}_{\pi}^{\text {mis }}(\mathrm{y})=\left\{\mathrm{x} \in \operatorname{Des}_{\pi}(\mathrm{y}) \mid \neg(\exists n \in N)\left(B S \pi^{n} x\right)\right\} \\
& \operatorname{Des}_{\pi}^{\text {pfex }}(\mathrm{y})=\left\{\mathrm{x} \in \operatorname{Des}_{\pi}(\mathrm{y}) \mid \operatorname{Card}\left(A s c_{\pi}(x)\right)>1\right\}
\end{aligned}
$$


And family $C h n_{\pi}(y)=\left\{C h n_{\pi}^{i}(y) \mid i \in I\right\}$ linear ordered chains directed to the base station:

$$
\left(\operatorname{Chn}_{\pi}^{i}(y) \subset \operatorname{Des}_{\pi}(y) \mid i \in I\right)(\mathrm{BS}=\perp \wedge \mathrm{y}=\mathrm{T})
$$

Where the symbol $\perp$ indicates the minimum in the set (i.e. base station), a symbol $\mathrm{T}$ indicates the maximum in the set (the node $y$ ) (Fig. 1 and Fig. 2).

\section{Modeling Adaptive Behavior in WSN}

\subsection{Actions and in WSN}

For modeling the activity adaptive WSN, we approach relational and define such basic concepts as well as action and behavior. The activity will be considered as network nodes activity, while preserving the activity from the perspective of external node. In addition, behavior is the result of activities of the group nodes on the network.

Network communication activities are described using three, dyadic relationships defined by the activities set Act. Activities are activities that have each of the nodes performing individually, however, depending on any other nodes, which are located in its vicinity. Ability to perform an action depends on the state, in a node (other actions will be carried out at the time of creating, other during normal network operation) and its end causes a change in the node status. Therefore, all actions which may take network nodes are described as a transformation of a Cartesian product of the WSN nodes set (Nodes) and a set of nodes state (States) The result is a new node, therefore, the Act is defined as ternary relation:

$$
\text { Act : Nodes } \times \text { States } \rightarrow \text { States }
$$

Examples of actions may be: measuring environmental parameters, data aggregation, sending data to a selected/all nodes or the reception of the data. Since the nodes are autonomous, each of them may act independently of the other nodes. This has an unquestionable advantage, because in this way, the WSN may also carry out a number of different operations. On the other hand, a number of actions makes perfect sense only when two or more nodes cooperate with each other in carrying out the activities related to each other (cooperate with each other). The node activity makes a lot of sense, if in its vicinity there is at least one node that performs an action - receiving. In this case, cooperation between the nodes is required to perform such that each of the nodes of remains with each other in defined relationships. We propose to do this with the relations of subordination $-\pi$, tolerance; $-\vartheta$ and collisions $-\chi$. Behavior of a collection of relations with each 
other, in certain cause-and-effect relations, (here we are using the relation $\pi, \vartheta$ and $\chi$ ), are temporary as well as spatial (neighbor relation).

On this basis we can build the product set Behavior (Beh), whose elements are equivalence classes of the relation $R$ :

$$
\text { Beh }: \text { Act } / R=\left\{a c t_{[x]} \in A c t \mid a c t_{[x]} R x\right\}
$$

The theoretical basis for the research activity (WSN) is the relational model [12] of cooperation between individual nodes on the network. This model describes the relations between the nodes and determining what action these nodes may take. Most of the advantages are in describing actions of individual nodes, which are autonomous components compatible, in order to achieve the objective defined globally for the entire network. Thus, the relational, not only contains in itself the existing solutions for communication routing, but you can also achieve global objectives through local action by individual network nodes in the WSN. Ultimately, it is a bridge between a locality and global behavior in the network, and thus between the actions of a group of behaviors of these elements.

\subsection{Design with Adaptive Behavior in the Neighborhood Node}

Routing multi-hop node cooperation is a key form of activity in the WSN [4, 21, 22]. Most nodes cannot communicate directly with the BS, because their range of radio communications is not sufficient. The WSN is a fully distributed system in which nodes with resources necessary for the implementation of calculations need to communicate with neighbors in order to maintain a compact state across network infrastructure. There are many WSN algorithms, which use some of the neighborhood concepts (e.g. neighborhood algorithms; multi-hop, reliable, bidirectional or geographic). We also we will use this concept, starting from its definition. Let $\operatorname{Map}(X, Y)$ is a mapping from $\mathrm{X}$ to $Y$ (surjection), where $\operatorname{Sub}(X)$ is defined as family all subsets of $X$. In the presented model the neighborhood $N$ can be expressed as follows:

$N \in \operatorname{Map}($ Nodes, Sub(nodes))

Let $N(k)$ will be neighbor of node $k$ and $N(S)$ will be neighbor of set nodes $S$ be defined as follows:

$$
\begin{aligned}
& \left.N(k)_{\mid k \in \text { Nodes }}=\left\{y \in \text { Nodes } \mid y R_{N} k\right)\right\} \\
& \left.N(S)_{\mid S \in \text { Nodes }}=\left\{y \in \text { Nodes } \mid(\exists x \in S) y R_{N} k\right)\right\}
\end{aligned}
$$

In the WSN there is present a natural localism, associated with the concept of a natural neighborhood. Both of these concepts are the result of radio coverage restrictions (mainly technical) or anisotropic propagation radio waves. Thus, the term, the natural locality occurs. Another locality type is introduced by 
clusterization. In this case, their locality is limited to the cluster and is type of simplification. It makes it easy to calculate, but at the same time, the potential solutions became narrowed. As a result, the natural locality is seen as the most appropriate approach, particularly from the perspective of a node. Formally, the natural neighborhood in the WSN is family indexed sets $N=\left\{\mathrm{N}_{\mathrm{i}} \mid \mathrm{i} \in \mathrm{I}\right\}$ and is globally characterized as follows:

$(\forall i \in I)\left(N_{i} \neq \emptyset\right) \cup N_{i}=$ Nodes

$$
(\exists i, j \in I \mid i \neq j)\left(N_{i} \cap N_{j} \neq \varnothing\right)
$$

From a local node point of view we obtain:

$$
(\forall y \in \text { Nodes })\left(\exists ! i \in I \mid y \in \cap N_{i} \neq \varnothing\right)
$$

Where the symbol $\exists^{!}$means "there are many". So, based on a relation (5.8) we can say that the defined neighbor relationship is not a partition of a set Node.

With a well defined neighborhood (5.6) - (5.8) we will try to make decomposition chains (4.5) for the various neighborhood nodes. The decomposition must be simple and identical for each of the nodes. After this, as nodes are scattered in the area, self organization of WSN nodes takes place. A set of nodes is not an ordered set and the main task is to establish a certain order. It supports the network communications further activity. The considerations made in the previous sections result that in the relationship of subordination $\pi$, which is transitive and reflexive one, define preorder. Unfortunately, this does not exclude loops in the process of building routing paths (Fig. 3, left). The possibility of loop occurrence is excluded due to presence of weak partial order through requirement that the relation $\pi$ was an anti-symmetric one (Fig. 3, right). Such an outcome is already a considerable success of self-organization. However, average energy expenditures in designing routing paths, are very high. A packet, not spread in the network indefinitely, but very often long circulates in the WSN before it reaches the BS|. Only linear ordered chains (4.5) are the expected final result (Fig. 4).
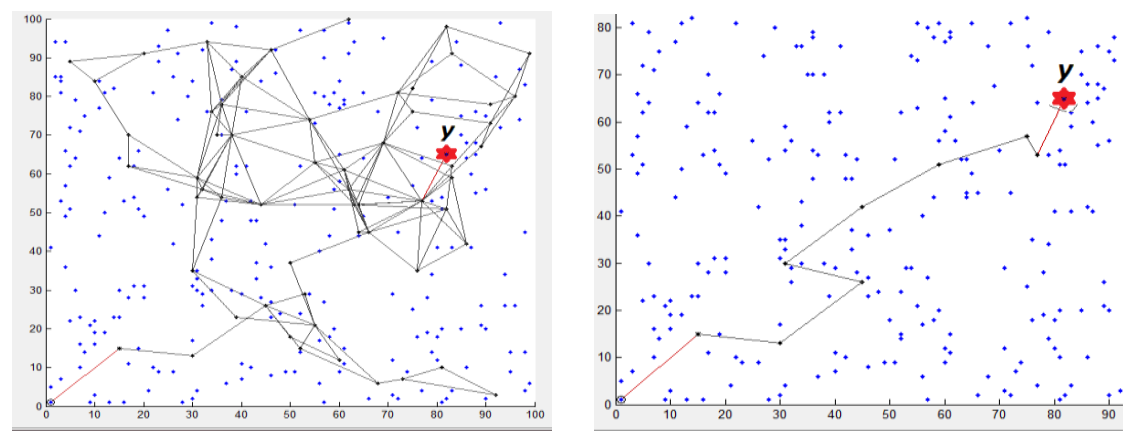

Figure 3

Sample tracks/chains of $\mathrm{N}(y)$ with preordered graph (left) and weak ordered partial graph (right) 
Before introducing a proper order let's look now at its familiar algorithm for determining distance from the base station [26]. This algorithm does not determine the Euclidean distance, but it specifies a minimum number of retransmissions, which must be carried out to ensure a packet submission to the BS. Procedure to be followed in broadcast mode is started by the BS sending the message "my distance from $B S$ is 0 ". Each node that receives a message "my distance from BS 0 " defines its status as "my distance from $B S$ is 1 " and the message is disseminated within the neighborhood. In general, a node that receives a message "my distance from $B S$ is $x$ ", determines their status as "my distance from $B S$ is $x+1$ ", then if it has received the message for the first time, broadcasts this message in its vicinity. The initial status of each node is 0 , allowed status operations are: an increase in status, when it is zero and a reduction in status, when it is non-zero. When this procedure is completed, each node with non-zero status $X+1$ can communicate with at least one node with status $X$. If a node has a status 0 , this means that it does not have any neighbors and may not participate in the WSN network activity. As a result, each $k$ node knows its distance to the base station, dish $(k)$ defined as the minimum number of retransmissions. More importantly, knows the distance all neighbors $\operatorname{dish}(x)$, where $x \in N(k)$ (Fig. 5).

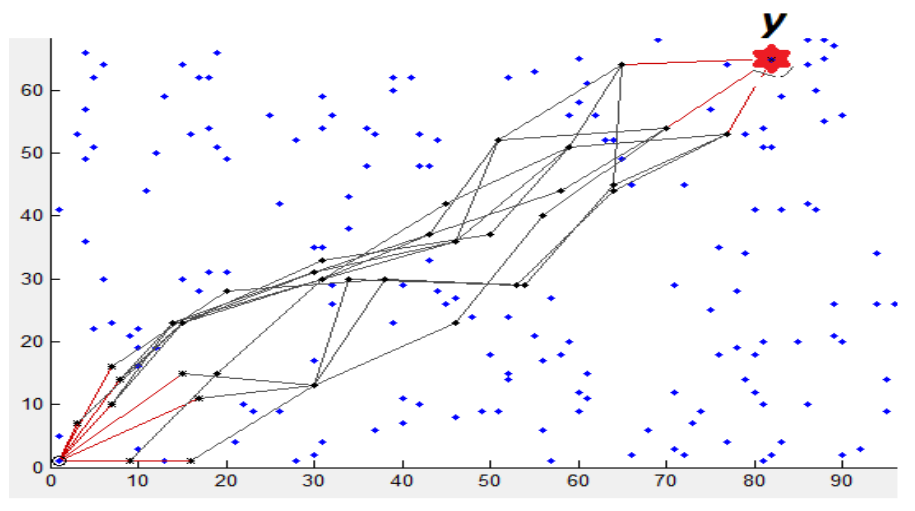

Figure 4

Sample tracks/chains of $\mathrm{N}(y)$ with the order line

On the basis of this information is possible to split neighborhood $N(k)$ into three subsets:

$$
\begin{aligned}
& N_{<}(k)=\left\{y \in N(k) \mid \operatorname{dis}^{h}(y)<\operatorname{dis}^{h}(k)\right\} \\
& N_{\leq}(k)=\left\{y \in N(k), y=k \mid \operatorname{dis}^{h}(y) \leq \operatorname{dis}^{h}(k)\right\} \\
& N_{>}(k)=\left\{y \in N(k) \mid \operatorname{dis}^{h}(y)>\operatorname{dis}^{h}(k)\right\}
\end{aligned}
$$

In considering the relationship of Subordination $(\pi)$ for any node $k \in \operatorname{Des}_{\pi}(y)$ its communication activity is limited only to its neighbors, i.e.: 
$N_{\pi}(k)=\{x \in N(k) \mid x \pi k\}$

Knowing $\mathrm{N}_{<}(\mathrm{k})$, which was set in the WSN, we intend to identify the components of the node set (5.8). Accept that $N_{\pi}(k) \subseteq N_{<}(k)$

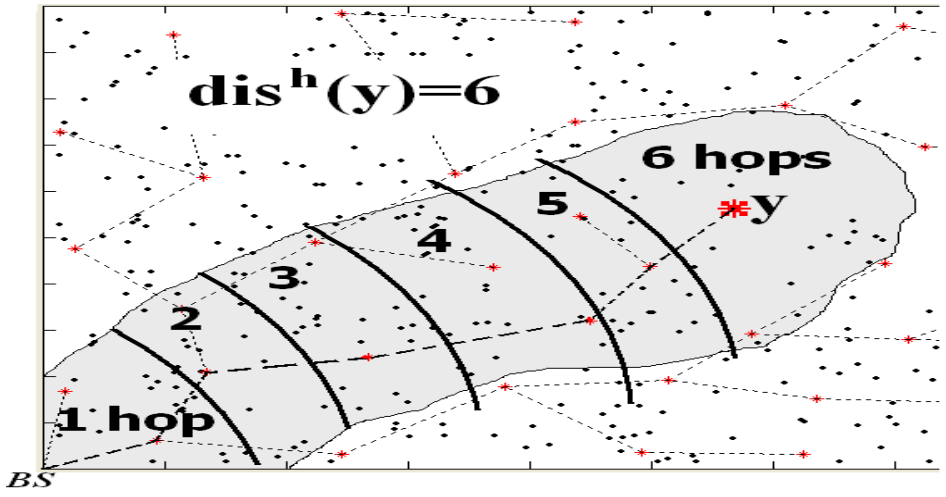

Figure 5

Determination of distance $\operatorname{dish}(s)$ from the base station $(B S)$ [17]

and it is sufficient that designated paths shall drift to the BS. Cardinality of the set $N_{<}(k)$ shall determine the maximum number of such possible paths. Moreover, each of these paths will be optimal in the Pareto sense. For instance in Figure 5: retransmission to the base station - there are seven relays, regardless of which path is selected. It is likewise with a tolerance relation $\vartheta$. For any node $k \in \operatorname{Des}_{\vartheta}(y)$ its communication activity is limited only to its neighbors, i.e.

$N_{\vartheta}(k)=\{x \in N(k) \mid x \vartheta k\}$

Knowing $N_{\leq}(k)$, which was set created in the WSN self organization process, identify the components of the set (5.11) for the node $k$. Accept that $N_{\vartheta}(k) \subseteq$ $N_{\leq}(k)$, But it does not guarantee that every simple path will strive to the BS. Tolerance is symmetrical and reflexive, and it is not enough to have a relation of the ordering. However, the tolerance and the subordination results on fuzzy effect of subordination, which is very important in situations of routing paths in areas of excessive noise. Of course, the sole relation tolerance is insufficient to guarantee the network cooperation sensors will ensure that messages are sent to the BS. Tolerance, however, has properties that make it very useful as secondary/complementary relation to the relation of Subordination. Especially when due to their locality effects, the packet becomes stuck in the blind localization (dead end). The relation of Subordination will not address this situation. The Tolerance relation is perfect and can handle it because of the symmetry (returning a packet to the last source) by proposing an alternative path. The relations of Subordination and Tolerance ensure that the message always reaches the BS, but at the same time allowing transmission to nodes tolerated to increase the number of available communication paths. 


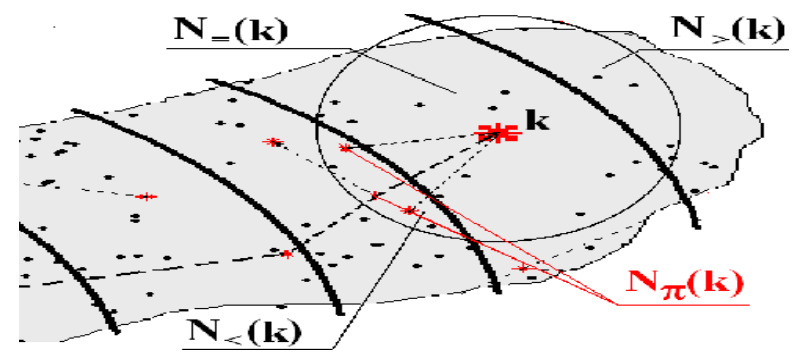

Figure 6

Building chains $C h n(k)$ in the neighborhood node $k$ [17]

As before, the maximum number of possible paths is- equal to the cardinality of the set $N_{\leq}(k)$ (Fig. 6)-

Third relation, the Collision $\mathcal{X}$, which for any node $k \in \operatorname{Des}_{\chi}(y)$, limited communication activity to neighbors:

$N_{\chi}(k)=\{x \in N(k) \mid x \chi k\}$

Knowing the $N_{>}(k)$, which was set in the WSN self organization process, we intend to identify the node components of the set (5.12). Let's us assume $N_{\chi}(k) \subseteq N_{>}(k)$, but because of Collision the package in all the paths will be directed away from the BS. A Collision is symmetrical and anti-reflexive, hence, may not be an ordering relationship. However, for nodes it is useful, because it allows for definition of a neighbors set, with which cooperation is undesirable. As in the previous two cases, the maximum number of possible paths is the cardinality of the set $N_{>}(k)$ (Fig. 7).

\subsection{Management of Adaptive Behaviors}

In the process, preceding the appropriate operation of the network, nodes of the network will learn about their neighborhood. Then, by using the globally defined metric dish $(s)$, as described in previous sections, each node orders its neighborhood, creating sets (5.9). Node remains only to realize the following assignments:

$N_{\pi}(k) \subseteq N_{<}(k) \quad ; \quad N_{\vartheta}(k) \subseteq N_{\leq(k)} \quad ; \quad N_{\chi}(k) \subseteq N_{>}(k)$

Sets $N_{\pi}(k), N_{\vartheta}(k), N_{\chi}(k)$ are respectively, subsets of sets $N_{<}(k)$, $N_{\leq}(k), N_{>}(k)$. The following questions appear: which node should determine all of them? Which and how many nodes adjacent to them belong to them? The first question answer is determined by node on the basis of globally defined objective (e.g. from the neighborhood are selected these nodes, which have the highest energy). The number of nodes that belong to the specific sets is determined globally and is being implemented by the base station $B S$. 


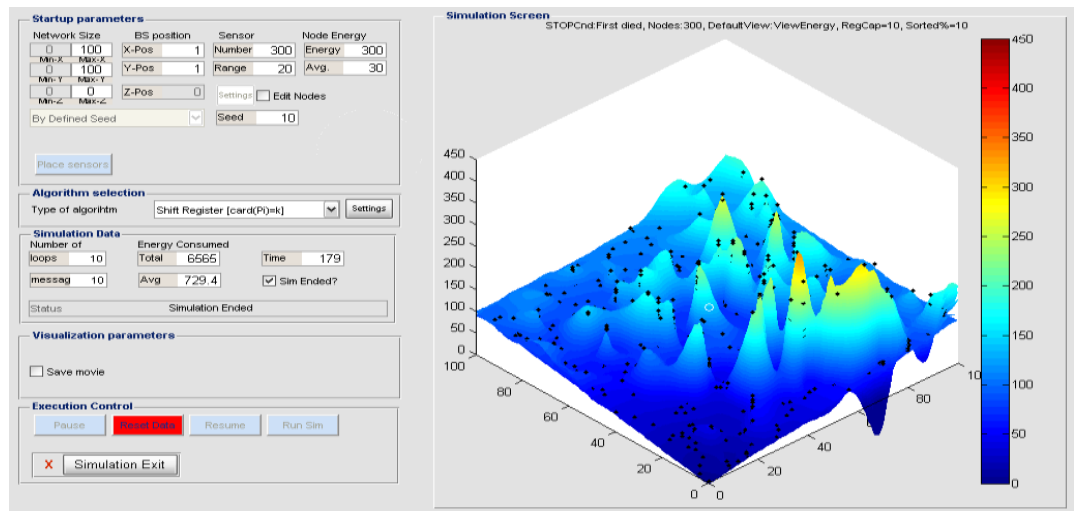

Figure 7

View simulator adaptive behavior with a locator, required globally by coefficients of relationship $\pi, \vartheta, \chi$

Globally, therefore, the main task of adaptive behavior management is to determine by the BS and send in the packet broadcast, required the number of sets $N_{\pi}(k), N_{\vartheta}(k), N_{\chi}(k)$. These are the cardinality of the set for individual subsets or percentage of cardinality of the set related to the cardinality of the number of neighborhoods (Fig. 7). Locally, on the basis of known resources neighbors, each node selects neighbors who will belong to specific subsets.

\section{Summary}

A relational approach provides a good presentation for adaptive collective behavior in a WSN. Quality of this tool is mainly due to the fact that it allows a consistent modeling of global and local phenomena. Locally means, in this approach, in the neighborhood. We also have neighborhood node (5.4), as is the other approaches, but we extend the concept of neighborhood to neighborhood nodes (set (5.5)). Assuming this set is a single set, these concepts are equivalent. Another extreme case is that, when a node for which neighborhood is the entire WSN. Then neighbors are also the entire WSN named global, and this is the case. Transition between these two extremes; local and global is smooth, because the cardinality of nodes set, for which we set neighborhood (5.5), can vary from 1 to the cardinality of Nodes WSN (all nodes). Partition to the local/global ceases to be dichotomous, moving from the alternative "or either' to 'this or that'.

Adaptive behavior is characterized accordingly:

- Locally, by selecting retransmitter, suitable to the prevailing ambient conditions. As a node retransmitting short extracts is the one that best meets the criterion, adopted centrally routing quality.

- Globally, routing path is modified, according to transmission constraints arising in the WSN. So, conversion to bypass routing areas of high noise. 
Group behavior is respectively:

- Locally, a relational approach gives the ability to use spatial routing. A node does not choose one retransmitter but indicates a recommended one to undertake these role nodes/neighbors. Groups indicated in this way, neighbors shall decide which of them receives a packet and forward it.

- Globally, the build routing path is the result of intentionally targeted individual nodes. None of the nodes (except last one) forming a routing path to the BS, is not able to send information to it. This is why group interaction between nodes, in the relational schema restrictions, gives a result achieved by nodes, which are not able to do it separately.

\section{Bibliography}

[1] Braginsky, D., Estrin, D, Rumor Routing Algorithm for Sensor Networks, Proc. of the $1^{\text {st }}$ Workshop on Sensor Networks and Applications, Atlanta, GA, 2002

[2] Brooks, R. R., Pirretti, M. Zhu, M. Iyengar, S. S., Distributed Adaptation Methods for Wireless Sensor Networks, Global Telecommunications Conference, GLOBECOM '03, 2003, Vol. 5, pp 2967-2971, DOI10.1109/GLOCOM.2003.1258778

[3] Burmester, M., Le T. V., Yasinsac, A., (2007) Adaptive for Protocols: Managing Security and Redundancy in Dense Ad Hoc Networks, Ad Hoc Networks, Volume 5, Issue 3, pp. 313-32

[4] Cohn, A. G. , Bennett, B. , Gooday, J. M., Gotts, N. M. , Representing and Reasoning with Qalitative Spatial Relations about Regions, Cohn, A. G., Bennett B., Gooday J. M., Gotts N M, eds. Spatial and Temporal Reasoning, Dordrecht, Kulwer, 1997, 97-134

[5] G. Coulouris, J. Dollimore, T. Kindberg, Distributed Systems: Concepts and Design, Addison-Wesley, June 2005. ISBN13:9780321263544

[6] Descartes R., L. J. Lafleur, Discourse on Method and Meditations, 1960, New York, the Liberal Arts press, ISBN10:0-672 -60278-4

[7] Fang O., Gao J., Guibas L. J., de Silva V., Zhang L., Glider: Gradient Landmark-based Distributed Routing for Sensor Networks, $24^{\text {th }}$ Annual Joint Conference of the IEEE Computer and Communications Societies. INFOCOM 2005, 13-17 March 2005, Vol. 1, pp. 339-350

[8] Jaroń, J., Systemic Prolegomena to Theoretical Cybernetics, Scient. Papers of Inst. of Techn. Cybernetics, Wroclaw Techn. Univ., No. 45, Wroclaw, 1978

[9] Manjeshwar, A., Agrawal, D. P., Teen: A Routing Protocol for Enhanced efficiency in Wireless Sensor Networks, $15^{\text {th }}$ International Parallel and Distributed Processing Symposium (IPDPS' 01), Parallel and Distributed Processing Symposium, International, 2001, Vol. 3, pp 30189, Sun courseware 
[10] Mills, Kevin L., A Brief Survey of Self-Organization in Wireless Sensor Networks, Wirel. Commun. Mob. comput., 2007, Vol. 7, pp. 1-12, Wiley InterScience, DOI: 10.1002/WCM.499

[11] Nikodem J., Autonomy and Cooperation as Factors of Dependability in Wireless Sensor Network, Proceedings of International Conference on Dependability of Computer Systems, Poland, June, 2008 / Eds Wojciech Zamojski, .Los Alamitos, IEEE Computer Society [Press], 2008, pp. 406413, http://dx.doi.org/10.1109/DepCoS-RELCOMEX.2008.50

[12] Nikodem J., Klempous R., Chaczko Z., Modeling of Immune Functions in a Wireless Sensor Network, 20 ${ }^{\text {th }}$ European Model. and Simul. Symp.. EMSS 2008, Campora S. Giovanni, Italy, 2008. pp. 45-50

[13] Nikodem J.: Designing Communication Space in Wireless Sensor Network Based on Relational Attempt, LNCS, Roberto Moreno-Díaz, Franz Pichler, Alexis Quesada-Arencibia (Eds.). Berlin, Springer, 2009, Vol. 5717, pp. 83-90, http://dx.doi.org/10.1007/978-3-642-04772-5_12

[14] Nikodem J., Modelling of Collective Animal Behaviour Using Relations and Set Theory /. Lecture Notes in Computer Science, ISSN 0302-9743; Vol. 8111Pt. 1 / Roberto Moreno-Díaz, Franz Pichler, Alexis QuesadaArencibia (eds.). Berlin; Heidelberg, Springer, 2013, pp. 110-117, http://link.springer.com/chapter/10.1007/978-3-642-53856-8_14

[15] Nikodem J., The Dilemma of Choice in Management of Communication Process in WSN / Lecture Notes in Computer Science, ISSN 0302-9743; Vol. 6927,Pt. 1 / Roberto Moreno-Díaz, Franz Pichler, Alexis QuesadaArencibia (eds.). Berlin: Springer, 2012. pp. 48-55, http://dx.doi.org/10.1007/978-3-642-27549-4

[16] Nikodem J., Modelling and Activity in Wireless Sensors Network / Lecture Notes in Computer Science, Roberto Moreno-Díaz, Franz Pichler, Alexis Quesada-Arencibia (eds.). Berlin, Springer, 2007, Vol. 4739, pp. 18-25

[17] Nikodem J., Klempous, R., Nikodem M., Chaczko, Z., Woda M., Directed Communication in Wireless Sensor Network Based on Digital Terrain Model, $2^{\text {nd }}$ Intern. Symp. on Logistics and Industr. Informatics, Sept. 2009, Linz, Austria. Piscataway, NJ: IEEE, 2009, s. 87-91

[18] Nikodem J., Klempous, R., Woda M., Chaczko Z.: Multihop Communication in Wireless Sensor Network Based on Directed Cooperation, Selected papers on Broadband Communication, Information Technology \& Biomedical Application, BroadBandCom'09, Technical University of Wroclaw, 2009, s. 239-241

[19] Nikodem J., Woda M., Nikodem M., Spatial Communication Activity in Wireless Sensor Networks Based on Migrated Base Stations, Wireless Sensor Networks Technology and Protocols, ed. by Mohammad A. Matin. Rijeka: InTech, 2012, pp. 99-116, http://www.intechopen.com/books/wireless-sensor-networks-technologyand-protocols 
[20] Sung-Min, J., Young-Ju, H., Tai-Myoung, C, 2007. The Concentric Clustering Scheme for Efficient Energy Consumption in the PEGASIS, $9^{\text {th }}$ International Conference on Advanced communication Technology, Vol. 1, page(s): 260-265

[21] Vaidya, D., Peng, J., Yang, L. , Rozenblit, J. W. (2005) A Framework for Sensor Management in Wireless and Heterogeneous Sensor Network, $12^{\text {th }}$ IEEE International Conference on the Engineering of Computer-Based Systems, pp. 155-162, April 2005, Greenbelt, MD, USA

[22] Vakil, S., Liang, B., Balancing Cooperation and interference in Wireless Sensor Networks, IEEE Comm. Society on Sensor and ad hoc Communications and Networks, Secon '06, Vol. 1, pp. 198-206

[23] Veyseh, M., Wei, B., Mi, N. F. (2005) An Information Management Protocol to Control Routing and Clustering in Sensor Networks, Journal of Computing and Information Technology - CIT 13 (1), pp. 53-68

[24] O. Younis, Fahmy S.: Heed: A Hybrid, Energy-Efficient, Distributed Clustering approach for ad hoc Sensor Networks, IEEE Transactions on Mobile Computing, October 2004 (Vol. 3, No. 4), pp. 366-379

[25] H. Kaur, S. Sharma; A Comparative Study of Wireless Technologies: Zigbee, Bluetooth LE, Enocean, Wavenis, Insteon and UWB; Proc. of the Intl. Conf. on Recent Trends In Computing and Communication Engineering, RTCCE 2013; Institute of Research Engineers and Doctors; ISBN: 978-981-07-6184-4; DOI:10.3850/ 978-981-07-6184-4_60

[26] Akyildiz F., W. Su, Sankarasubramaniam Y., and Cayirci E, Wireless Sensor Networks: a Survey, Computer Networks, March 2002, Vol. 38, No. 4, pp. 393-422

[27] Szymanski J., Chaczko, Z. C., Rodanski, B., Enabling Design and Development of Wireless BANs using 802.15.x Standards, in Moreno Diaz, Roberto; Pichler, Franz; Quesada Arencibia, Alexis (eds), LNCS, Eurocast 2013, Springer-Verlag, Las Palmas de Gran Canaria, Spain, 2013

[28] C. Feng, L. Yang and J. W. Rozenblit, Adaptive Tracking in Energy Sensitive Distributed Wireless Sensor Networks, Ad Hoc and Sensor Wireless Networks, 12(1-2), pp. 55-77, 2011

[29] Yang, C. Feng, J. Peng, and J. W. Rozenblit, "A Multi-modality Framework for Energy Efficient Tracking in Large Scale Wireless Sensor Networks," Proc. of the $2^{\text {nd }}$ IEEE International Conference on Networking, Sensing and Control, pp. 916-921, Ft. Lauderdale, FL, USA, April 2006

[30] L. Yang, C. Feng, J. W. Rozenblit and H. Qiao, "Adaptive Tracking in Distributed Wireless Sensor Networks," Proc. of the $13^{\text {th }}$ IEEE Intl. Conference and Workshops on the Engineering of Computer Based Systems (ECBS' 06), pp. 103-111, Potsdam, Germany, March 2006 